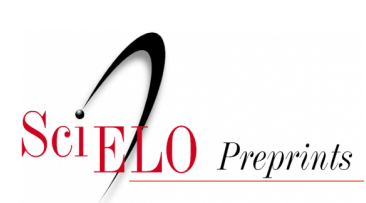

Situação: O preprint não foi submetido para publicação

\title{
Mortalidade por COVID-19 no Brasil: uma análise do Registro Civil de óbitos de janeiro de 2020 a fevereiro de 2021
}

Mauro Sanchez, Erly Moura, Juliano Moreira, Rodrigo Lima, Ivana Barreto, Claudia Pereira, Leonor Santos

https://doi.org/10.1590/SciELOPreprints.2012

Este preprint foi submetido sob as seguintes condições:

- O autor submissor declara que todos os autores responsáveis pela elaboração do manuscrito concordam com este depósito.

- Os autores declaram que estão cientes que são os únicos responsáveis pelo conteúdo do preprint e que o depósito no SciELO Preprints não significa nenhum compromisso de parte do SciELO, exceto sua preservação e disseminação.

- Os autores declaram que a pesquisa que deu origem ao manuscrito seguiu as boas práticas éticas e que as necessárias aprovações de comitês de ética de pesquisa estão descritas no manuscrito, quando aplicável.

- Os autores declaram que os necessários Termos de Consentimento Livre e Esclarecido de participantes ou pacientes na pesquisa foram obtidos e estão descritos no manuscrito, quando aplicável.

- Os autores declaram que a elaboração do manuscrito seguiu as normas éticas de comunicação científica.

- Os autores declaram que o manuscrito não foi depositado e/ou disponibilizado previamente em outro servidor de preprints ou publicado em um periódico.

- O autor submissor declara que as contribuições de todos os autores estão incluídas no manuscrito.

- O manuscrito depositado está no formato PDF.

- Os autores declaram que caso o manuscrito venha a ser postado no servidor SciELO Preprints, o mesmo estará disponível sob licença Creative Commons CC-BY.

- Caso o manuscrito esteja em processo de avaliação ou sendo preparado para publicação mas ainda não publicado por um periódico, os autores declaram que receberam autorização do periódico para realizar este depósito.

Submetido em (AAAA-MM-DD): 2021-03-20

Postado em (AAAA-MM-DD): 2021-03-22 
Mortalidade por COVID-19 no Brasil: uma análise do Registro Civil de óbitos de janeiro de 2020 a fevereiro de 2021.

Mortality from COVID-19 in Brazil: analysis of death's civil registry from 2020 January to 2021 February.

Mortalidade por COVID-19 no Brasil

Mauro Niskier Sanchez, maurosanchez@unb.br, http://orcid.org/0000-0002-0472-1804, Departamento de Saúde Coletiva, Faculdade de Ciências da Saúde, Universidade de Brasília. Brasília, Distrito Federal, Brasil.

Erly Catarina de Moura, erlycm@gmail.com, http://orcid.org/0000-0002-9237-432X, pesquisadora CNPq, Brasília, Distrito Federal, Brasil.

Juliano Ribeiro Moreira, juliano.ribeiro@ expertise.net.br, http://orcid.org/0000- 0002-46826073, Expertise Inteligência e Pesquisa de Mercado Ltda, Belo Horizonte, MG, Brasil.

Rodrigo Tobias de Sousa Lima, tobiasrodrigo@gmail.com, https://orcid.org/0000-00024098-5276, Laboratório de História, Políticas Públicas e Saúde na Amazônia, ILMD/Fiocruz Amazônia, Amazonas, Brasil.

Ivana Cristina de Holanda Cunha Barreto, ivana.barreto24@gmail.com, http://orcid.org/0000-0001-8447-3654, Fundação Oswaldo Cruz, Eusébio, Ceará, Brasil.

Claudia Cristina de Aguiar Pereira, cpereira@ensp.fiocruz.br, https://orcid.org/0000-00031389-9214. Departamento de Administração e Planejamento em Saúde, Escola Nacional de Saúde Pública Sergio Arouca (ENSP/Fiocruz), Rio de Janeiro, RJ, Brasil.

Leonor Maria Pacheco Santos, leopac@ unb.br, https://orcid.org/0000-0002-6739-6260,

Departamento de Saúde Coletiva, Faculdade de Ciências da Saúde, Universidade de Brasília, Brasília, DF, Brasil 
Correspondência para: Mauro Niskier Sanchez, maurosanchez@unb.br, Departamento de Saúde Coletiva, Faculdade de Ciências da Saúde, Universidade de Brasília. Brasília, Distrito Federal, Brasil.

Os autores declaram não haver conflito de interesse.

Estudo financiado pela Chamada Pública MCTI/CNPq/CT-Saúde/MS/SCTIE/Decit No 07/2020

Aprovação do CEP - Esta investigação dispensa avaliação de Comitê de Ética, pois se encontra de acordo com o artigo primeiro, "pesquisa que utilize informações de domínio público", da Resolução do Conselho Nacional de Saúde (CNS) nº 510, de 7 de abril de 2016.

Contribuição dos autores: Mauro Niskier Sanchez: Escrita do manuscrito, ideia original, revisão na extração e seleção dos dados e revisão final do manuscrito. Erly Catarina de Moura: Escrita do manuscrito, ideia original, extração dos dados e revisão final do manuscrito. Juliano Ribeiro Moreira: extração dos dados e revisão final do manuscrito. Rodrigo Tobias de Sousa Lima: Escrita de parte do manuscrito e revisão final. Ivana Cristina de Holanda Cunha Barreto: Escrita de parte e revisão crítica do manuscrito. Claudia Cristina de Aguiar Pereira: Escrita de parte do manuscrito e revisão final. Leonor Maria Pacheco Santos: Escrita de parte do manuscrito, ideia original e revisão final. Todos os autores revisaram e aprovaram a versão final do manuscrito. 


\section{Resumo}

Objetivo: analisar a evolução da mortalidade por COVID-19 no período de janeiro de 2020 a fevereiro de 2021 no Brasil. Métodos. Dados sobre todos os óbitos foram obtidos do Portal da Transparência, alimentado continuamente pelos cartórios civis do país. Foi considerado óbito por COVID-19 quando havia citação de COVID-19, coronavírus ou novo coronavírus como causa de óbito. As taxas de mortalidade por COVID-19 foram padronizadas por sexo e faixa etária, conforme estimativa populacional para 2020. Mortalidade por COVID-19 proporcional foi calculada por faixa etária e região, conforme o sexo. Mortalidade geral proporcional foi calculada por local de falecimento e região, conforme causa do óbito. Resultados. Dos 1.596.130 óbitos registrados, $16 \%$ tiveram COVID-19 como causa básica, a taxa de mortalidade no Brasil foi de 119,9 por 100 mil habitantes, chegando a 410,5 em Roraima para o sexo masculino. Altas taxas foram encontradas principalmente na região Norte e as menores na região Nordeste. A maior proporção foi entre 70 e 79 anos de idade. A morte por COVID-19 em domicílio atingiu 3,8\% dos óbitos na região Norte e 3,4\% no Nordeste. Conclusões. O uso de dados do registro civil é de grande importância para o monitoramento atualizado da mortalidade por COVID-19, demonstrando que o país enfrenta, em 2021, aumento de óbitos e agravamento da pandemia

Palavras-chave: Mortalidade, COVID-19, coronavírus, epidemiologia, pandemia, Brasil. 


\section{Abstract}

Objective: to analyze the evolution of mortality due to COVID-19 in the period from January 2020 to February 2021 in Brazil. Methods. Data on all deaths were obtained from the transparency portal, continuously fed by the country's civil registry offices. It was considered death by COVID-19 when there were citations of COVID-19, coronavirus or new coronavirus in the cause of death. Mortality rates for COVID-19 were standardized by sex and age group, according to a population estimate for 2020. Proportional COVID-19 mortality was calculated by age group and region, according to sex. General proportional mortality was calculated by place of death and region, according to the cause of death. Results. Of the 1,596,130 deaths recorded, $16 \%$ had COVID-19 as a basic cause, the mortality rate in Brazil was 119.9 per 100 thousand inhabitants, reaching 410.5 in Roraima for men. High rates were found mainly in the North and lowest in the Northeast. The highest proportion was between 70 and 79 years of age. Death by COVID-19 at home reached 3.8\% of deaths in the North and 3.4\% in the Northeast. Conclusions. The use of civil registry data is of great importance for a timely monitoring of COVID-19 mortality, thus showing the country faces, in 2021, an increase in deaths, worsening the pandemic.

Keywords: Mortality, COVID-19, coronavirus, epidemiology, pandemic, Brazil. 


\section{INTRODUÇÃO}

Após um ano do aparecimento do primeiro caso registrado de COVID-19, o Brasil passou a ser segundo país com o maior número de óbitos no mundo ${ }^{1,2}$ e a historicidade do contágio precisa ser revista para melhor entender os motivos deste complexo cenário.

A primeira morte, segundo o Ministério da Saúde ${ }^{3}$, ocorreu em São Paulo no dia 17 de março, 20 dias após o primeiro caso confirmado no Brasil. A pandemia evoluiu e alcançou o pico da primeira onda epidemiológica da doença em 29 de julho de 2020, registrando o total de 1590 óbitos em um único dia ${ }^{1}$. Seja pelo recrudescimento da pandemia ou o advento de uma mutação do SARS-CoV2 ${ }^{4}$, uma segunda onda do número de casos e óbitos assolou o país, iniciando no mês de novembro de 2020 e mantendo sua tendência de crescimento até o começo de março com o registro de 2286 óbitos em 24 horas ${ }^{1}$. É notório que ocorreram o agravamento simultâneo do aumento do número de casos e óbitos com a sobrecarga de hospitais ${ }^{5}$. No Brasil existem estatísticas oficiais sobre mortes que representam opções de informações confiáveis para entender a dinâmica do coronavírus; muitas são originários do setor saúde (Ministério da Saúde e Fiocruz), mas existem também os registros cartoriais de óbitos, que constituem uma fonte necessária para análises no âmbito da saúde pública.

No Brasil, o Conselho Nacional de Justiça (CNJ) foi criado em $2004^{6}$, tendo como função o controle externo do Judiciário. Embora composto em sua maioria por magistrados, a participação popular garante relativa transparência administrativa e processual, especialmente no que se refere aos recursos humanos e financeiros, informatização e gestão de informações. Neste contexto, em 2018 foi criado o portal da Transparência do Registro Civil ${ }^{7}$, sob a responsabilidade da Associação Nacional dos Registradores de Pessoas Naturais, que permite o acesso a dados sobre nascimentos, casamentos e óbitos, obtidos diretamente dos cartórios de Registro Civil, conforme a Unidade da Federação. Ainda, no âmbito da transparência, o CNJ, 
em 2020, passou a contabilizar, com atualização diária, os óbitos pelo coronavírus, contando com a iniciativa dos Cartórios de Registro Civil do Brasil. O prazo legal para a informação do óbito pela família ao Cartório é de 24 horas, que deve fazer o registro em até cinco dias e, depois, enviar para Central Nacional de Informações do Registro Civil em até oito dias, para disponibilização no portal. Desta forma, o portal da transparência se configura como uma ferramenta ágil para o entendimento de óbitos relacionados à COVID-19 na esfera nacional. O uso de fonte de informação alternativa, ainda pouco explorada em estudos científicos, permite o conhecimento oficial do perfil dos óbitos que poderiam ter sido tratados em hospitais, preenchendo as lacunas de informações sobre contagens oficiais que precisam ser alimentadas com regularidade diária e de forma transparente ${ }^{7}$. Para o enfrentamento ao coronavírus, é preciso entender o peso da COVID-19 no perfil da mortalidade do país, para que estudos científicos possam orientar a tomada de decisão dos gestores no sentido de adoção de medidas não farmacológicas que proteja o sistema de saúde e a vida das pessoas. Portanto, intenta-se elucidar como a mortalidade por coronavírus reagiu com o tempo, quais idades e sexo mais afetados nas Unidades Federativas, bem como o seu perfil com enfoque na observação dos óbitos domiciliares no período da pandemia. Neste sentido, o presente estudo tem como objetivo analisar a evolução da magnitude e gravidade da mortalidade por COVID-19 no período compreendido das duas ondas atualmente registradas no Brasil.

\section{MATERIAL E MÉTODOS}

Trata-se de um estudo transversal ou seccional com abordagem analítico-descritiva e quantitativa sobre a evolução dos picos de óbitos por COVID-19 no Brasil. Os dados referentes aos óbitos de $1^{\circ}$ de janeiro de 2020 a 28 de fevereiro 2021 foram obtidos, no formato CSV, no dia 05 de março de $2021^{8}$, e reconstruídos individualmente, contemplando as seguintes 
variáveis: região geográfica (Norte, Nordeste, Sudeste, Sul ou Centro-Oeste), Unidade da Federação (UF), sexo (masculino ou feminino), faixa etária (<10, 10-19, 20-29, 30-39, 40-49, 50-59, 60-69, 70-79, 80-89, 90-99 ou $\geq 100$ anos de idade), causa do óbito (COVID-19, síndrome respiratória aguda grave (SRAG), insuficiência respiratória (IR), pneumonia, septicemia, outras causas e causas mal definidas), óbito por COVID-19 (não ou sim) e local do óbito (setor saúde, domicílio, via pública ou outro).

Para este estudo, considerou-se o registro da doença COVID-19 como causa básica confirmada ou suspeita de coronavírus segundo a Declaração de Óbito. SRAG, IR, pneumonia e septicemia foram consideradas causas relacionadas ao coronavírus, pela semelhança da clínica. O local do óbito registrado como hospital, outros serviços de saúde, posto de saúde, ambulância ou Serviço de Atendimento Móvel de Urgência (SAMU) foram agrupados como "local de saúde", outro local, ou local ignorado foram agrupados como "outro", "domicílio" e "via pública” foram mantidos individualizados.

O número de óbitos foi contabilizado por sexo e faixa etária conforme UF. Foram calculadas as taxas de mortalidade por COVID-19 para 100 mil habitantes, padronizada por sexo e por faixa etária $(<10,10-19,20-29,30-39,40-49,50-59,60-69,70-79,80-89$ ou $\geq 90$ anos de idade), tendo a estimativa populacional do Instituto Brasileiro de Geografia e Estatística para o país no ano $2020^{9}$, como padrão. Mortalidade proporcional dos óbitos relacionados à COVID-19 foi calculada por faixa etária e região para ambos os sexos. Mortalidade proporcional por COVID19, por causas relacionadas à COVID-19 e por outras causas foram tabuladas por local de falecimento e região.

O processo para a determinação de COVID-19 como causa de óbito obedece a um modelo hierárquico, isto é, se houver citação na declaração de óbito: COVID-19, Coronavírus ou Novo Coronavírus, como causa básica, considera-se COVID-19 (suspeita ou confirmada) ${ }^{8}$. Foram 
também analisadas outras causas de óbito, entre as quais doenças respiratórias como SRAG, IR, pneumonia e septicemia.

\section{RESULTADOS}

Foram avaliados 1.596.130 registros, sendo 1.364 .744 referentes ao ano 2020 e 231.386 ao ano 2021. No total, $0,4 \%$ dos registros estavam sem informação sobre o sexo e $1,4 \%$ sobre a faixa etária. No grupo de óbitos por COVID-19, a falta de informação sobre sexo e sobre faixa etária foi da ordem de 0,04 e $0,2 \%$, respectivamente.

Os óbitos por COVID-19, no período avaliado, representaram 16,0\% do total de mortes no país (Figura 1), variando de $13,5 \%$ no Nordeste a $22,7 \%$ no Norte. O percentual de óbitos por doenças respiratórias potencialmente associadas ao coronavírus foi de 33,2\%, com menores percentuais nas regiões Norte $(30,9 \%)$ e Nordeste $(31,8 \%)$.

A taxa de mortalidade por COVID-19 (Figura 2) variou de 62,1 óbitos por 100 mil habitantes no Maranhão a 354,0 em Roraima, sendo sempre maior entre o sexo masculino, chegando a 410,5 em Roraima para este sexo. De modo geral, observam-se altas taxas nos estados do Norte, com a região apresentando coeficiente de 174,9 mortes por 100 mil habitantes, seguida pela região Centro-Oeste $(150,9)$, Sudeste $(128,8)$, Sul $(99,5)$ e a mais baixa no Nordeste $(95,9)$. O Brasil apresentou taxa de 119,9, sendo 138,0 para o sexo masculino e 102,2 para o feminino. As cinco UFs com as maiores taxas por 100 mil habitantes foram: Roraima (354,0), Amazonas (298,1), Acre (218,7), Amapá (218,6), e Rondônia (213,5), todas da região Norte. O Brasil apresentou taxa global de 119,9, sendo 138,0 para o sexo masculino e 102,2 para o feminino. A taxa de mortalidade por COVID-19, embora maior para o sexo masculino, mostra diferenças quanto à faixa etária (Figura 3), sendo maior no sexo masculino entre 40 e 79 anos de idade e no feminino após 79 anos. Observa-se, para ambos os sexos, que o pico se dá entre 70 e 79 anos 
de idade. Destaca-se, a região Norte com maior percentual de óbitos nas menores idades. Quanto ao local do óbito, a maioria se deu no setor saúde (Figura 4), tanto para as causas relacionadas à COVID-19, quanto às demais causas, sendo que a região Centro-Oeste teve menos de $90 \%$ das mortes no setor saúde. Todavia chama a atenção, o alto percentual de mortes domiciliares por causas relacionadas à COVID-19 na região Nordeste $(12,8 \%)$, bem como por outras causas, que chega a atingir 39,3\% na região Nordeste, ficando em 31,6\% para o país. Quanto à distribuição regional, os maiores percentuais de mortes domiciliares por COVID-19, foram observadas nas regiões Norte $(3,8 \%)$ e Nordeste $(3,4 \%)$. Salienta-se, ainda, a região Centro-Oeste com maior registro de mortes por COVID-19 em outro local (9,2\%).

De um modo geral, o Brasil enfrenta em 2021 aumento de óbitos e agravamento da pandemia, como pode ser visto na Figura 5. Nota-se claramente duas ondas epidemiológicas. Destaca-se, que houve um aumento significativo do número de óbitos registrados em janeiro e fevereiro de 2021.

\section{DISCUSSÃO}

O banco de dados utilizado neste estudo propiciou uma análise rápida da situação da pandemia no país e em cidades com mais de 50 óbitos registrados por COVID-19, mas limitou a obtenção direta de dados por determinados períodos, à exceção do número de óbitos. Os óbitos são agregados diariamente e totalizados para o ano respectivo sem menção de data de ocorrência. Deste modo, mesmo com dados coletados em 05 de março de 2021, se assumiu a possibilidade da não completude do banco de dados do ano 2020 por conta de eventuais atrasos de registro, fora do prazo legal determinado. Todavia, os dados apresentados são corroborados por outros sistemas de monitoramento e por alguns poucos trabalhos que vem sendo publicados. 
Atualmente, existem no Brasil quatro fontes de informações contínuas sobre óbitos por COVID-19: o Portal Coronavírus Brasil do Ministério da Saúde, o MonitoraCovid-19 da Fiocruz e o Sistema de Informação de Vigilância da Gripe (SIVEP-Gripe), que também incorpora dados sobre mortes por COVID-19 e SRAG e o Sistema de Registro Civil do CNJ (utilizado neste estudo). Há uma pequena diferença entre eles, explicada por critérios próprios de cada provedor. Todavia, todos eles alertam para a grave situação sanitária que assolou o país. Somente após a divulgação de dados do Sistema de Informações sobre Mortalidade (SIM) do Ministério da Saúde, padrão-ouro, será possível avaliar a sensibilidade de cada sistema. Além disto, uma análise mais detalhada propiciará melhor entendimento da mortalidade pela COVID19, ainda que a padronização por sexo e faixa etária elimine vieses relacionados à estrutura populacional, não contempla outros fatores próprios da dinâmica populacional, sanitários e sociais, como relatam alguns autores ${ }^{10-12}$. Outra questão é ser considerada é a possibilidade de subnotificação de óbitos e de diagnóstico impreciso ${ }^{13}$.

Estudo realizado por Silva et al $^{14}$, utilizando SIVEP-Gripe, desde o início da pandemia até o final de janeiro do corrente ano, destaca a região Norte com maior aumento das taxas de mortalidade por COVID-19, após padronização por faixa etária, sendo que a capital do estado do Amazonas, com a capital apresentando taxa de 412,5 por 100 mil habitantes. O mesmo achado foi observado no nosso estudo quanto à região Norte, porém o estado com maior coeficiente foi Roraima. Esta diferença pode ser explicada pelo tempo de coleta de dados, uma vez que avançamos até início de março, quando os registros de novos casos originais da segunda onda estavam ainda em ascensão, associado ao fato da segunda onda ter iniciado primeiramente no Amazonas. Cumpre ressaltar que a região Norte é a que mais sofre com as iniquidades sociais e assistenciais no Brasil. 
Comparando-se nosso estudo com aquele realizado por Heuveline e Tzen ${ }^{15}$ sobre o primeiro semestre de 2020, nota-se que, pelas Unidades Federativas selecionadas e a população padrão utilizada para ajuste das taxas de mortalidade para COVID-19 naquele trabalho, o Rio de Janeiro permanece à frente das demais UFs, porém o estado de Goiás sobe da quinta para segunda posição, o Ceará cai da segunda para a terceira, São Paulo permanece na quarta, Pará sobe da sexta para a quinta e Pernambuco cai da terceira para a sexta. Estas diferenças podem ser explicadas, principalmente, pelo período de análise dos dados, além de outros fatores populacionais não computados no nosso estudo, como expectativa de vida, e a potencialidade de transmissão do coronavírus. Ainda assim, os dados atuais mostram que o país enfrenta uma das piores situações mundiais de casos de COVID-19.

Adicionalmente, a mortalidade por COVID-19 se iniciou homogeneamente em todas as regiões e mais precocemente na região Norte. O percentual de população economicamente ativa que perdeu a vida pela doença agrava mais ainda a situação de miséria destas populações. Em Rondônia observou-se maior letalidade entre idosos, homens e pessoas pardas e $\operatorname{pretas}^{16}$.

Estudo desenvolvido no Reino Unido ${ }^{17}$, aponta diferenças regionais, com maior densidade nas áreas mais populosas, como as capitais, como acontece também no Brasil. Os autores relatam a associação entre o risco de óbito por COVID-19, associado ao sexo e à idade, às comorbidades próprias do envelhecimento e do sexo. Infelizmente, o sistema utilizado não fornece dados sobre comorbidades, de modo que esta análise não foi feita, mas evidenciou-se padrões distintos conforme sexo e idade. Este tema merece aprofundamento, o que somente será possível após a liberação do banco de dados do SIM.

Outro achado deste estudo, que merece destaque, é o percentual de óbitos ocorridos em domicílio, que no país, para todas as causas foi da ordem de 20,8\%. Neste caso, o local do óbito pode ser considerado como proxy da disponibilidade de assistência sanitária e hospitalar, 
afetando principalmente as pessoas portadoras de outras doenças, que não a COVID-19. Em 2019, o $\operatorname{SIM}^{18}$ já registrava 19,6\% de óbitos em domicílio, variando de 16,0\% no Sudeste a $26 \%$ no Nordeste. Já, o alto percentual de óbitos em outro lugar pode ser má qualidade do preenchimento da Declaração de Óbito, que se concentrou principalmente na região CentroOeste.

De fato, artigo publicado nos Arquivos Brasileiros de Cardiologia em $2020^{20}$ relatou um aumento no número de óbitos por parada cardiorrespiratória no Brasil apresentando como comorbidade clínica hipertensão arterial sistêmica para $22 \%$ dos casos investigados pela pesquisa. Este padrão de mortalidade provavelmente indica que pessoas portadoras de hipertensão arterial e outras doenças cardiovasculares deixaram de demandar os serviços de saúde em função da COVID-19, seja pela superlotação dos serviços, seja por medo de contrair o Sars-Cov2. Um estudo realizado com dados de Minas Gerais identificou que houve aumento nas mortes domiciliares por pneumonia, insuficiência respiratória, síndrome respiratória aguda grave, sepse, infarto agudo do miocárdio, acidente vascular cerebral e por outras causas cardiovasculares comparando-se 2020 ao mesmo período em $2019^{21}$. Outro estudo focado em algumas capitais brasileiras no primeiro semestre de 2020, já apontava elevado percentual de mortes em domicílios ou em vias públicas, especialmente em Manaus²2.

Em um cenário de poucos arranjos institucionais formais estabelecidos para a cooperação entre os estados brasileiros, o Consórcio Nordeste, criado em 2019, é uma iniciativa que ganhou relevância por sua atuação no momento da crise sanitária gerada pelo COVID-19 A sua ação tentou suprir, em parte, a falta de recursos e de coordenação do governo federal. São exemplos a cobrança pelos governadores do Nordeste, do provimento de insumos e equipamentos, da força de trabalho, de políticas públicas como a extensão do Programa Mais Médicos, das Parcerias de Desenvolvimento Produtivo para criar linhas de ação específicas voltadas para o 
enfrentamento da pandemia ${ }^{23}$. Contribui para tal, a criação de um Comitê Científico de alto nível em março de 2020, que ofereceu ao Nordeste e a todo o Brasil um exemplo em que " $a$ ciência de ponta foi incorporada ao arsenal de combate da pandemia de COVID-19 por um grupo de gestores públicos, gerando um raio de esperança para todos os brasileiros que discordaram frontalmente da atitude negacionista, incompetente e irresponsável adotada pelo governo federal durante toda a pandemia de COVID-19"24. Certamente este contexto contribuiu para a mitigação da crise na região, tanto assim que apesar dos recursos tradicionalmente mais escassos, o Nordeste apresentou a menor taxa de mortalidade por COVID-19 entre as regiões brasileiras, mesmo as com mais recursos hospitalares como o Sudeste e o Sul.

Conclui-se que a mortalidade do COVID-19 nos estados brasileiros demonstra ser parâmetro importante para compreender o padrão epidêmico que revelou as desigualdades regionais no acesso aos serviços especializados de saúde que coincidem com as regiões brasileiras mais vulneráveis socioeconomicamente. Oportunamente, é sugestivo que o risco diferenciado de morte nas diferentes localidades refere-se à dimensão potencial de disseminação, a estratificação descrita discrimina locais com densidades populacionais maiores, com maior grau de urbanização e maior desigualdade de renda, o que significa dizer que são locais com maior concentração absoluta e relativa de pessoas $^{25}$. A heterogeneidade de cobertura de saúde em termos de infraestrutura física (respiradores, leitos de UTI) e de profissionais de saúde resulta em um risco diferenciado de óbito nas diferentes localidades ${ }^{26}$. O Brasil enfrenta, neste início do ano de 2021, a fase mais grave da pandemia de COVID-19 e, provavelmente, ainda não atingiu o pico da segunda onda epidemiológica. Como decorrência assiste-se, com grande preocupação, ao possível colapso do sistema de saúde. 


\section{REFERÊNCIAS}

1. Brasil. Painel COVID-19 no Brasil. [internet]. 2020 [acessado em 2 mar. 2021]. Disponível em: https://susanalitico.saude.gov.br/extensions/covid-19 html/covid-19 html.html.

2. WHO. WHO Coronavirus Disease (COVID-19) Dashboard. [internet]. 2020 [Acesso em 2 mar. 2021]. Disponível em: https://covid19.who.int/table.

3. Brasil. Ministério da Saúde. 2020. Situação epidemiológica da COVID-19 [Internet]. 2020 [acessado em 2 mar. 2021]. Disponível em:

https://portalarquivos.saude.gov.br/images/pdf/2020/April/12/2020-04-11-BE9-Boletim-doCOE.pdf.

4. Naveca F, Souza V, Costa C, Nascimento V, Corado A, Nascimento F, et al. COVID-19 epidemic in the Brazilian state of Amazonas was driven by long-term persistence of endemic SARS-CoV-2 lineages and the recent emergence of the new Variant of Concern P. [Preprint]. Res Sq [Internet]. 2021; 1-21. Available from: https://www.researchsquare.com/article/rs275494/v1. https://doi.org/10.21203/rs.3.rs-275494/v1 (no prelo).

5. Noronha KVMS, Guedes GR, Turra CM, Andrade MV, Botega L, Nogueira D, et al. Pandemia por COVID-19 no Brasil: análise da demanda e da oferta de leitos hospitalares e equipamentos de ventilação assistida segundo diferentes cenários. Cad Saude Publica. 2020; 36 (6): e00115320. https://doi.org/10.1590/0102-311X00115320.

6. Conselho Nacional de Justiça. Criação do Conselho Nacional de Justiça [Internet] 2020 [acessado em 2 mar. 2021]. Disponível em: https://www.cnj.jus.br/sobre-o-cnj/quem-somos/.

7. Associação dos Registradores de Pessoas Naturais (ARPEN). Portal da Transparência Registro Civil. [Internet] 2020 [acessado em 2 mar. 2021]. Disponível em: https://transparencia.registrocivil.org.br. 
8. Associação dos Registradores de Pessoas Naturais (ARPEN). Portal da Transparência Registro Civil. [Internet] 2020 [acessado em 5 mar. 2021]. Disponível em: https://transparencia.registrocivil.org.br/especial-

covid\#: :text=A\%20fam\%C3\%ADlia\%20tem\%20at\%C3\%A9\%2024h,)\%2C\%20que\%20atu aliza\%20esta\%20plataforma)

9. Brasil. DATASUS. Tecnologia da informação a serviço do SUS. [Internet] 2020 [acessado em 2 mar. 2021]. Disponível em: http://tabnet.datasus.gov.br/cgi/tabcgi.exe?ibge/cnv/projpopuf.def

10, Hallal PC. Worldwide differences in COVID-19-related mortality. Ciênc saúde coletiva 2020, 25(Supl.1): 2403-2410. https://doi.org/10.1590/1413-81232020256.1.11112020.

11. Hallal PC e Hartwig FP, 2020; Estrutura etária e mortalidade por COVID-19. Ciênc saúde coletiva 2020, 25 (9): 3691. https://doi.org/10.1590/1413-81232020259.21182020.

12. Queiros BL, Freire FHMA, Lima EEC, Gonzaga MR. O papel da estrutura etária na análise da mortalidade por Covid-19. Cien Saude Colet 2020; 25 (9): 3689-3690. https://doi.org/10.1590/1413-81232020256.1.11112020.

13. Prado MF, Antunes BBP, Bastos LSL, Peres IT, Silva AAB, Dantas LF et al. Análise da subnotificação de COVID-19 no Brasil. Rev Bras Ter Intensiva 2020; 32 (2): 224-228. https://doi.org/10.5935/0103-507X.20200030

14. Silva GA, Jardim B, Lotufo P. Mortalidade por COVID-19 no Brasil ajustada por idade: mais alta na região Norte. https://doi.org/10.1590/SciELOPreprints.1874

15. Heuveline P, Tzen M. Beyond deaths per capita: comparative COVID-19 mortality indicators. BMJ Open 2021; 11: e042934. doi:10.1136/bmjopen-2020-042934.

16. Escobar AL, Rodriguez TDM, Monteiro JC. Letalidade e características dos óbitos por COVID-19 em Rondônia: estudo observacional. Epidemiol e Serviços Saúde [Internet]. 
2021;30(1). Available from: http://www.scielo.br/scielo.php?script=sci_arttext\&pid=S2237$\underline{96222021000100300 \& t \operatorname{lng}=p t)}$

17. Clough HE, McIntyre KM, Patterson GE, Harris JP, Rushton J. Use of routine death and illness surveillance data to provide insight for UK pandemic planning: lessons from COVID19. BMJ Open 2021; 8; 11 (2): e044707. https://doi.org/10.1136/bmjopen-2020-044707. PMID: 33558359; PMCID: PMC7871230

18. Brasil. DATASUS. Tecnologia da informação a serviço do SUS. [Internet] 2020 [acessado em 5 mar. 2021]. Disponível em: http://tabnet.datasus.gov.br/cgi/tabcgi.exe?sim/cnv/obt10uf.def

19. Silva GA, Jardim, BC, Santos CVB. Excesso de mortalidade no Brasil em tempos de COVID-19. Cien Saude Colet 2020; 25 (9): 3345-54. https://doi.org/10.1590/1413$\underline{81232020259.23642020 .}$.

20. Guimarães NS, Carvalho TML, Machado-Pinto J, Lage R, Bernardes RM, Peres ASS, Raposo MA et al. Aumento de óbitos domiciliares devido a parada cardiorrespiratória em tempos de pandemia de COVID-19. Arq Bras Cardiol 2021; 116 (2): 266-71. https://doi.org/10.36660/abc.20200547.

21. Alves T, Souza T, Silva S, Ramos N, Oliveira S. Análise de óbitos domiciliares e hospitalares por causas respiratórias e cardiovasculares durante a pandemia da COVID-19 em Minas Gerais. Visa em Debate, 2020; 8(3):104-113 https://doi.org/10.22239/2317-269x.01726. 22. Orellana JDY, Cunha GM, Marrero L, Moreira RI, Leite IC, Horta BL. Excesso de mortes durante a pandemia de COVID-19: subnotificação e desigualdades regionais no Brasil. Cad Saúde Pública 2020; 36 (1): e00259120. https://doi.org/10.1590/0102-311X00259120. 
23. Fernandez M, Pinto HA. Estratégia intergovernamental de atuação dos estados brasileiros: o Consórcio Nordeste e as políticas de saúde no enfrentamento à Covid-19. Revista Saúde em Redes 2020; 6 (S2): 7-21. https://doi.org/10.18310/2446-4813.2020v6n2p7-21

24. Nicolelis, Miguel AL. O Comitê Científico de Combate ao Coronavírus do Consórcio Nordeste e a pandemia de COVID-19 no Brasil. In: CONASS. Planejamento e Gestão. Coleção COVID-19, v2. Brasília, DF: Conselho Nacional de Secretários de Saúde, 2021. p. 126-45. 25. Guimarães RM, Araújo Eleutério T, Costa Monteiro-da-Silva JH. Estratificação de risco para predição de disseminação e gravidade da Covid-19 no Brasil. Rev Bras Estud Popul 2020; 28; (37): 1-17. https://dx.doi.org/10.20947/S0102-3098a0122.

26. Moreira RS. COVID-19: unidades de terapia intensiva, ventiladores mecânicos e perfis latentes de mortalidade associados à letalidade no Brasil. Cad Saúde Pública 2020; 36 (5): e00080020. https://doi.org/10.1590/0102-311x00080020. 


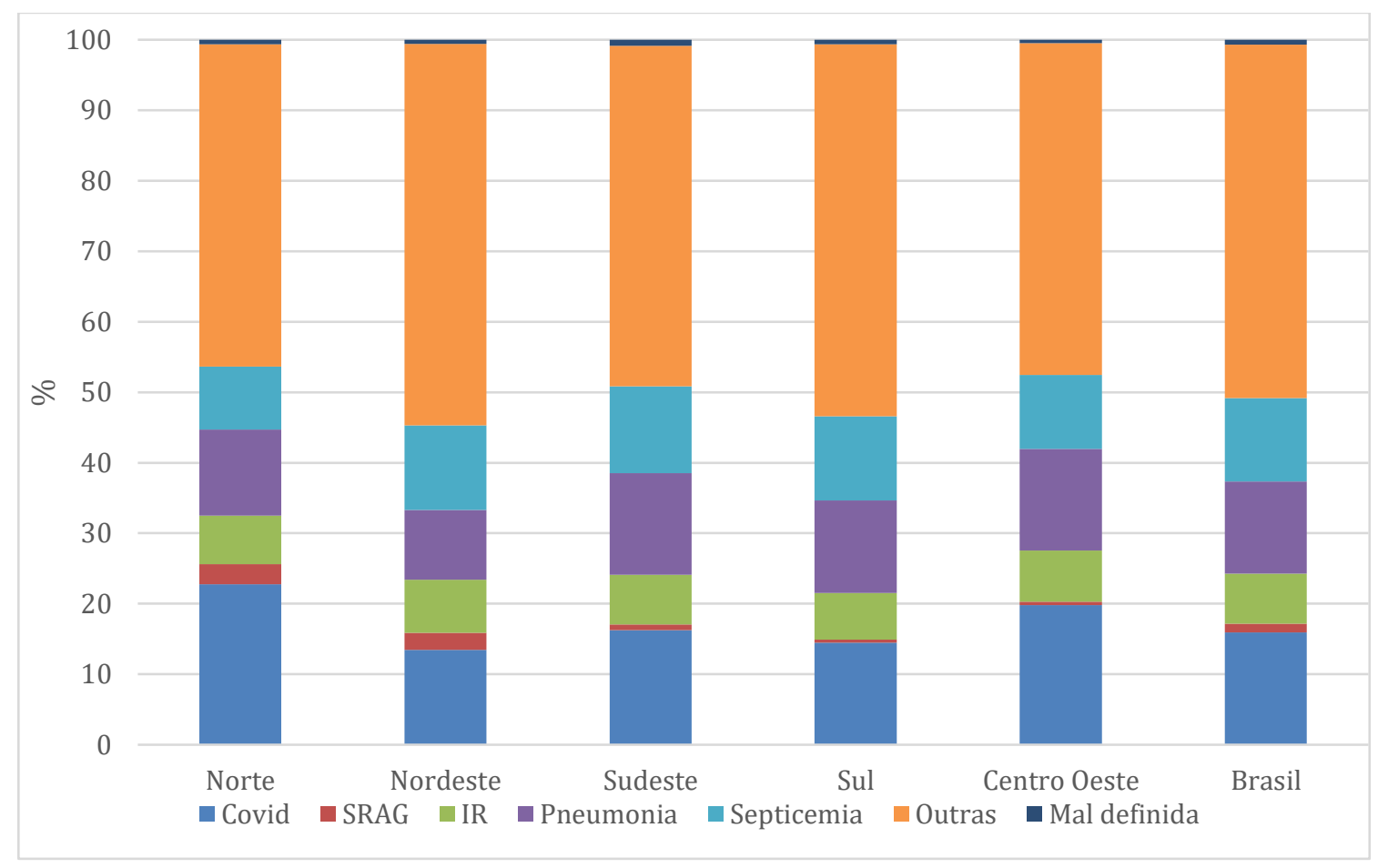

Figura 1 - Distribuição proporcional dos óbitos conforme causa e região, Brasil, 2020, Brasil, 2020 e 2021.

Fonte: https://transparencia.registrocivil.org.br/especial-covid

SRAG = síndrome respiratória aguda grave; $\mathrm{IR}$ = insuficiência respiratória 


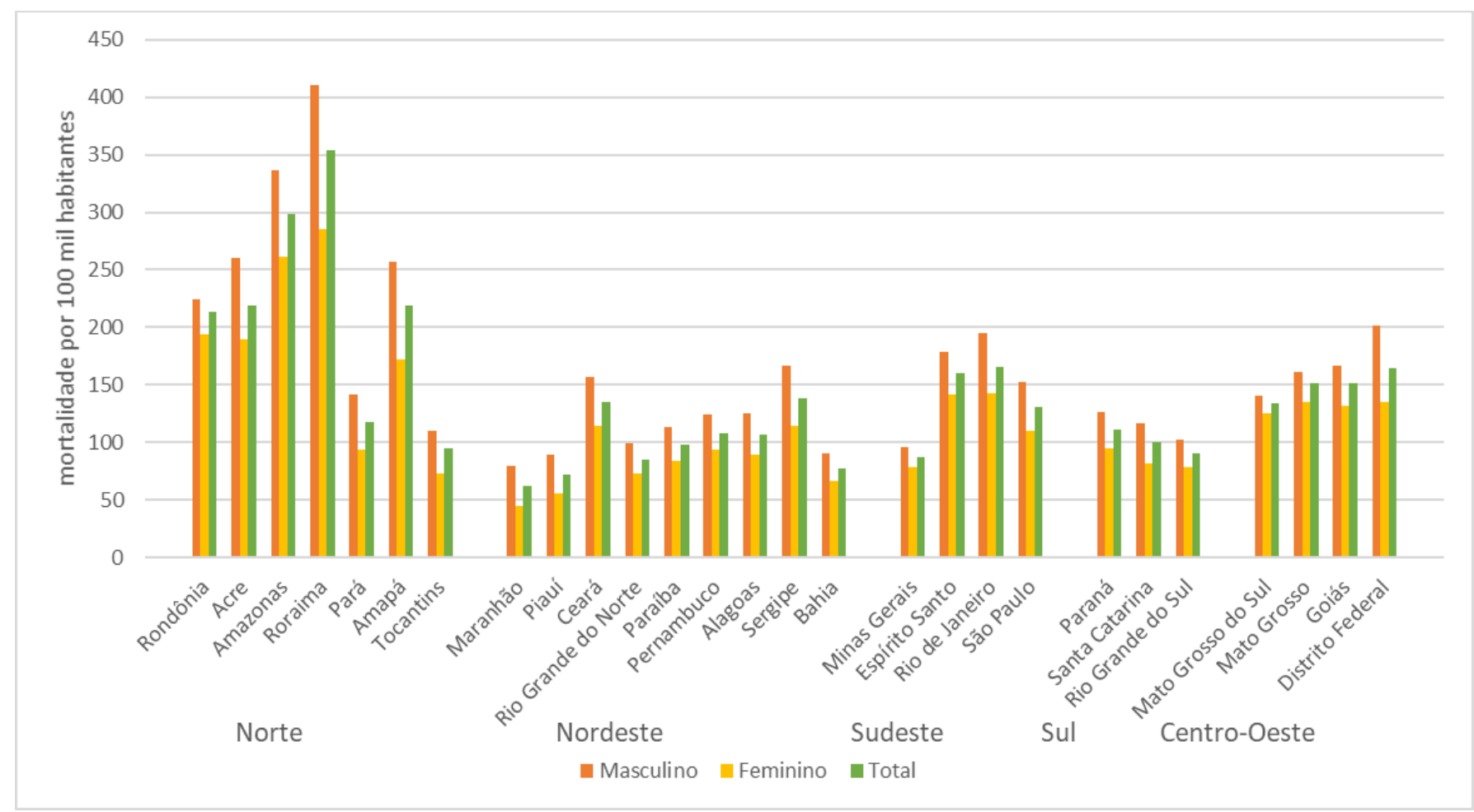

Figura 2- Taxa de mortalidade, padronizada por sexo e faixa etária, por 100 mil habitantes segundo sexo e Unidade da Federação, Brasil, 2020 e 2021.

Fonte: https://transparencia.registrocivil.org.br/especial-covid 


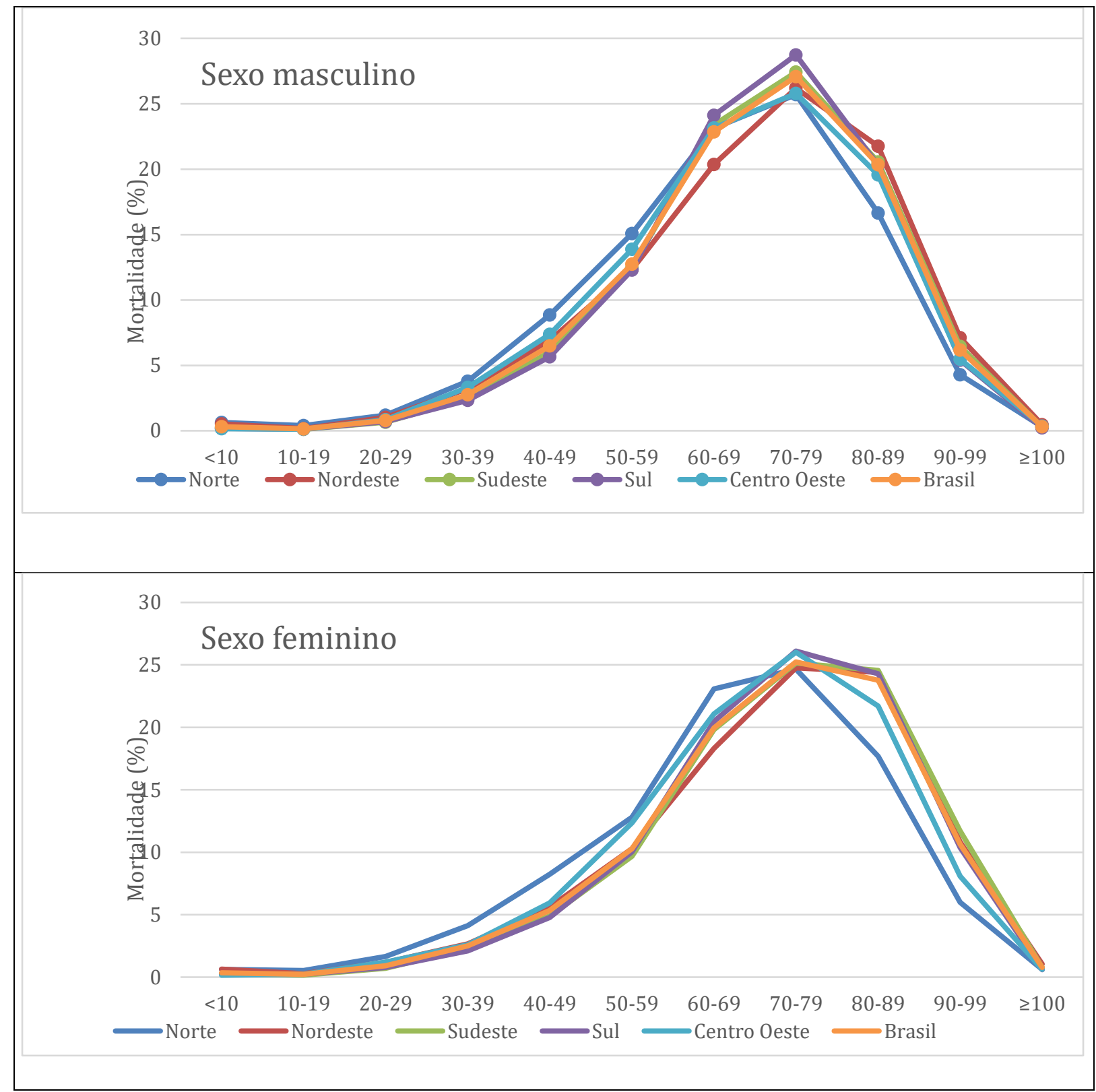

Figura 3 - Mortalidade por COVID-19 proporcional por faixa etária por região para o sexo masculino e feminino, Brasil, 2020 e 2021.

Fonte: https://transparencia.registrocivil.org.br/especial-covid 


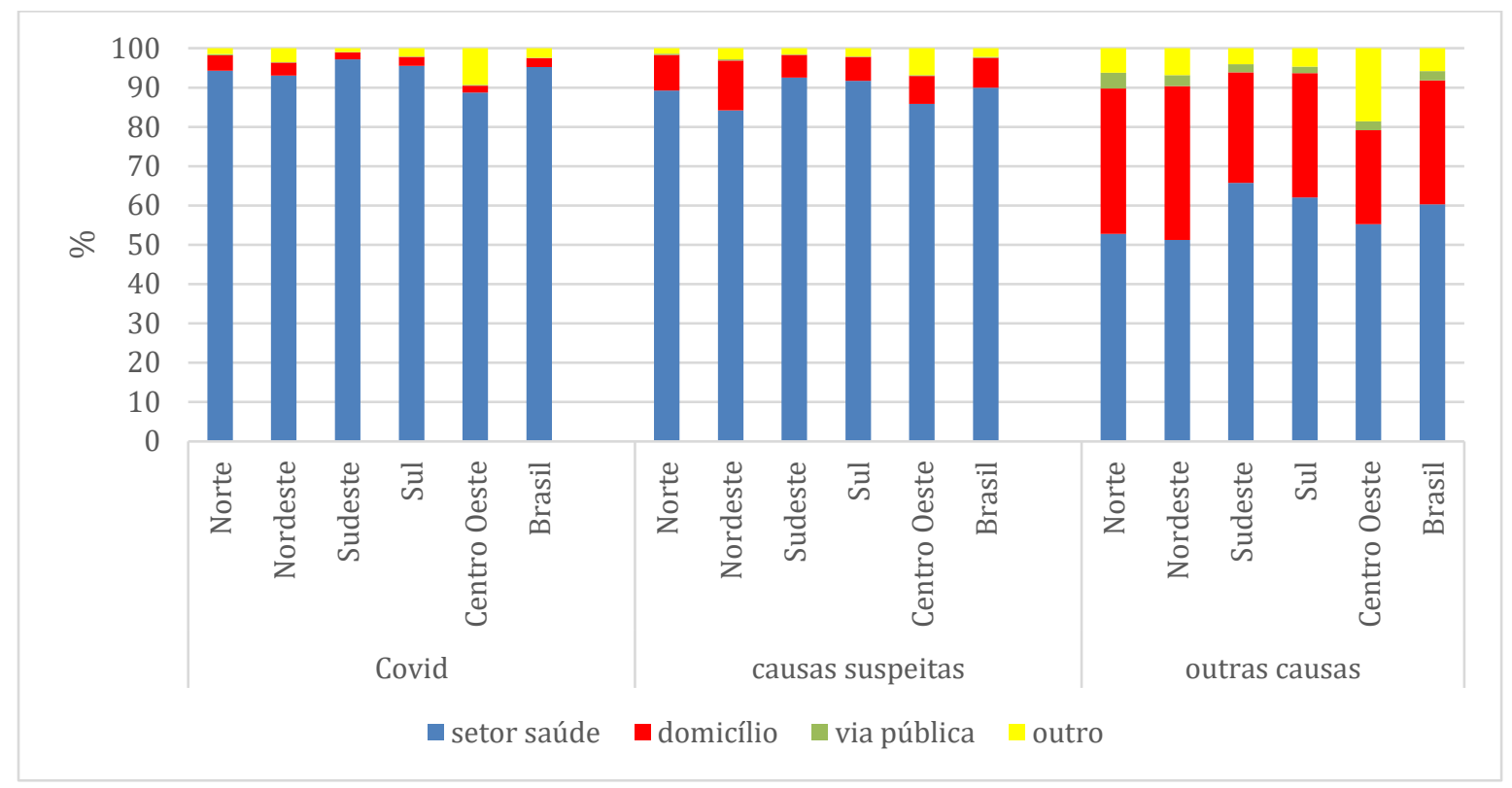

Figura 4 - Distribuição proporcional dos óbitos por COVID-19 e por outras causas conforme local do falecimento e região, Brasil, 2020 e 2021, Brasil, 2020.

Fonte: https://transparencia.registrocivil.org.br/especial-covid 


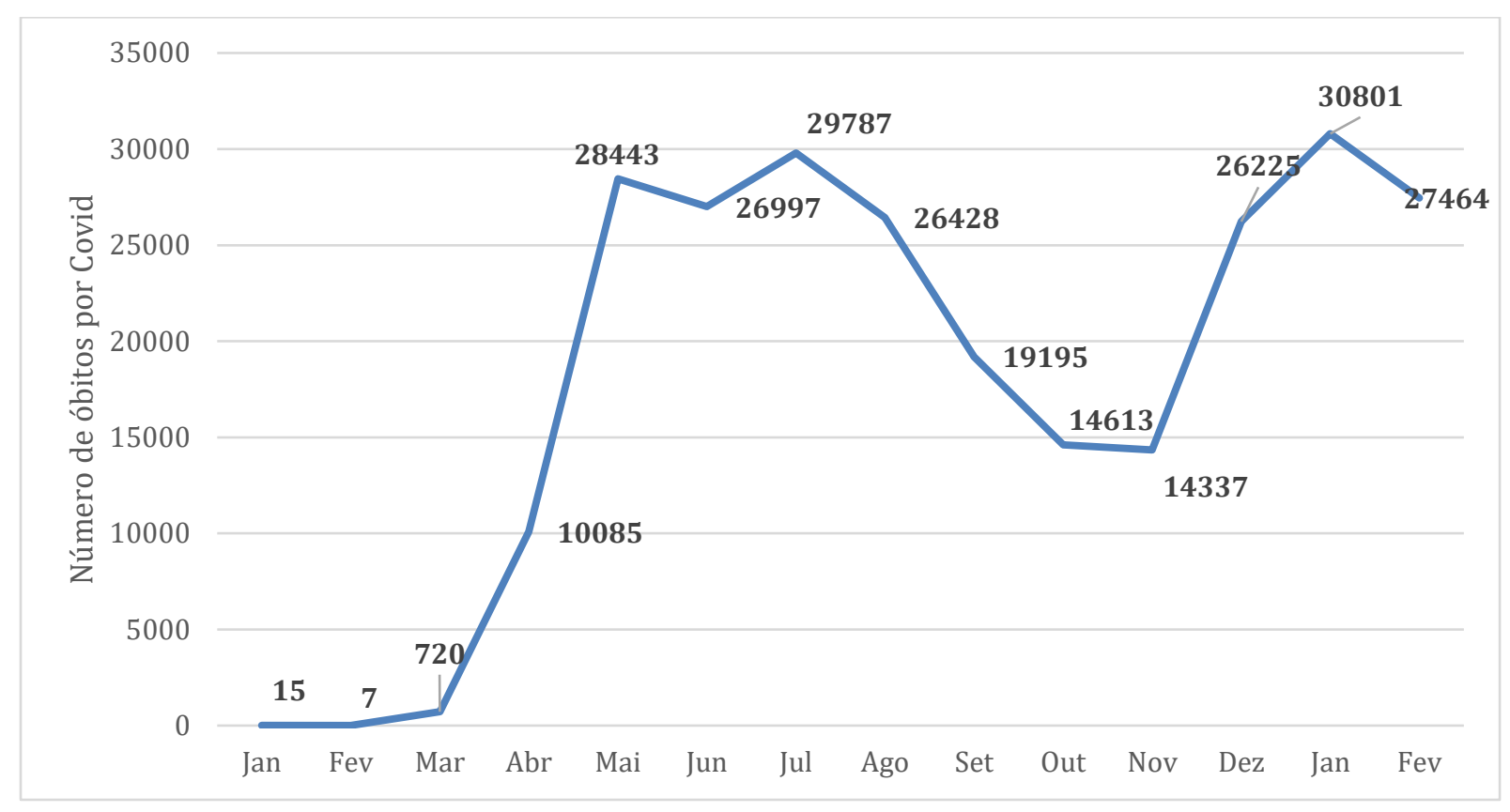

Figura 5 - Evolução do número de óbitos por COVID-19 segundo o mês de ocorrência, Brasil, 2020 e 2021.

Fonte: https://transparencia.registrocivil.org.br/especial-covid 